\title{
Adherencia al tratamiento y efectividad de un programa cognitivo conductual sobre la rumiación y expresión de la ira
}

\author{
Raúl J. Alcázar-Olán, Nicole Merckel-Niehus, Daniela Toscano-Barranco, Omar Barrera-Muñoz y \\ María Fernanda Proal-Sánchez
}

Universidad Iberoamericana Puebla, Puebla, México

Treatment adherence and effectiveness of a cognitive behavioral program on rumination and anger expression

\begin{abstract}
This research assessed the effects of a cognitive behavioral intervention in a group of 30 individuals with anger and rumination problems. Two groups were compared. The first group of participants attended seven or more sessions (high attendance), while the second group attended less than seven sessions (low attendance). Only the group with high attendance showed statistically significant changes in variables such as revenge, angry afterthoughts, angry memories, and understanding the causes of anger. However, both groups improved in the same proportion, according to the strong effect sizes. Participants who attended less than seven sessions reported that they missed sessions due to academic reasons (doing homework). Participants with high attendance reported they showed up to sessions due to their achievements obtained with the treatment.
\end{abstract}

Keywords: Adherence; anger; cognitive behavioral therapy; dropout; rumination.

Resumen: Esta investigación evaluó los efectos de una intervención cognitivo conductual grupal en 30 individuos con problemas de rumiación e ira. Se compararon dos grupos. El primero se formó de participantes que asistieron siete o más sesiones (alta asistencia), mientras que en el segundo los participantes asistieron menos de siete sesiones (baja asistencia). Se observaron mejoras estadísticamente significativas únicamente en el grupo con alta asistencia en variables de venganza, pensamientos posteriores a la ira, memorias de ira y entendimiento de las causas de la ira. Sin embargo, ambos grupos mejoraron en la misma proporción, lo cual se reflejó en tamaño del efecto fuerte. Los participantes que asistieron menos de siete sesiones informaron que su ausencia se debió a motivos académicos (hacer tareas). Los participantes con alta asistencia informaron que las razones para presentarse a las sesiones fueron los logros que obtenían con el tratamiento.

Palabras clave: Adherencia; ira; terapia cognitivo conductual; abandono; rumiación.

\section{Introducción}

La ira es una emoción intensa que tiene efectos negativos cuando se sale de control. La tendencia a sentir ira de manera rápida y frecuente se denomina alta ira rasgo (Spielberger y Reheiser, 2009). El término rasgo alude a una característica crónica, relativamente estable de sen-

Recibido: 05 de julio 2017; aceptado: 19 de diciembre 2017

Correspondencia: Raúl J. Alcázar-Olán, Departamento de Ciencias de la Salud, Universidad Iberoamericana Puebla, Blvd del Niño Poblano, 2901 Puebla, México.

Correo-e: raul.alcazar@iberopuebla.mx

Agradecimientos: A la Universidad Iberoamericana Puebla por su apoyo financiero para investigación. tir, pensar y comportarse de forma iracunda, en cualquier situación y en cualquier momento (Deffenbacher, 1992). La alta ira rasgo se ha asociado de manera consistente con diversos problemas incluyendo problemas de salud (Schum, Jorgensen, Verhaeghen, Sauro y Thibodeau, 2003), acoso cibernético (Wang, Yang, Yang, Wang y Lei, 2017), menor control de impulsos (Wilkowski y Robinson, 2007), y psicopatologías como ansiedad, depresión, estrés postraumático y dependencia a drogas o alcohol (Kassinove y Tafrate, 2006; Stimmel, Rayburg, Waring y Raffeld, 2005). La alta ira rasgo se encuentra asociada a diversos trastornos psicológicos de personalidad incluyendo el trastorno paranoide, límite, esquizoide, antisocial, narcisista, histriónico y dependiente (Cas- 
tilho, Xavier, Pinto-Gouveia y Costa, 2015; Kassinove y Tafrate, 2006).

Los tratamientos cognitivo-conductuales han mostrado de forma consistente su utilidad para enseñar a controlar la ira (Beck y Fernandez, 1998; Novaco, 1976, 1978). La recuperación abarca del 67 al $80 \%$ de los pacientes, mientras que el 33\% de las personas sin tratamiento mejoran (Beck y Fernandez, 1998; Gorenstein, Tager, Shapiro, Monk, y Sloan, 2007). Como resultado del tratamiento, las personas mantienen un adecuado control de su ira durante meses o incluso años posteriores (DiGiuseppe y Tafrate, 2003).

La manera en que tradicionalmente se han evaluado los resultados de un tratamiento psicoterapéutico es analizando los cambios estadísticamente significativos o con el tamaño del efecto, el cual indica la magnitud de los cambios (Cárdenas y Arancibia, 2014). Estos métodos han enfatizado la comparación de datos de forma grupal (p.ej., con apoyo de medias y desviación típica). Sin embargo, estos métodos tienen importantes limitaciones evaluando cambios individuales. Jacobson y Truax (1991) crearon el índice de cambio clínico confiable para superar esta limitación. Este índice fue construido para evaluar si, después de un tratamiento, el individuo pertenece o no a la población normal y cuánto cambió debido a la terapia. A pesar de su relevancia, encontramos sólo un estudio que utilizó este índice en el tratamiento de la ira, con resultados mostrando que entre el 40 y el $50 \%$ de los pacientes alcanzaron el índice de cambio clínico confiable (Deffenbacher, 2006).

Aunque el enfoque cognitivo conductual ha sido útil en el tratamiento de la ira (Beck y Fernandez, 1998), rara vez se ha evaluado si los pacientes se muestran satisfechos con el tratamiento. Esto es fundamental porque los tratamientos suelen incluir distintos tipos de técnicas y es posible que algunas sean más atractivas que otras para los participantes. Además, la satisfacción es relevante porque indica el grado en que las personas juzgan la importancia y validez social del tratamiento (Carter, 2010).

La rumiación de la ira consiste en mantener pensamientos o ideas alrededor de esta emoción. En particular, la rumiación de la ira se define como los procesos cognitivos recurrentes y no intencionales que surgen durante y después de un episodio de ira (Sukhodolsky, Golub y Cromwell, 2001). Los estudios han mostrado que la rumiación es perdurable y se asocia con efectos negativos. Por ejemplo, se ha encontrado asociación entre rumiar sobre transgresiones interpersonales y altos niveles de cortisol (Denson, Fabiansson, Creswell y Pedersen, 2009). También se ha encontrado que la rumiación persiste durante meses, al mismo tiempo que permanecen altos niveles de ira rasgo, lo cual refleja mayor frus- tración y tendencia a suprimir la ira (Takebe, Takahashi y Sato, 2016). En otro estudio (Izadpanah, Schumacher, y Barnow, 2017), la rumiación de la ira persistió durante cinco años, tiempo en el cual se presentó con mayores niveles de depresión, ansiedad y agresión.

A pesar de las consecuencias negativas de la rumiación de la ira, no encontramos estudios que se hayan enfocado en implementar un tratamiento para disminuir la rumiación de la ira. Además, su tratamiento puede servir como un parámetro adicional para evaluar la eficacia del tratamiento cognitivo conductual de la ira. Con base en que el tratamiento cognitivo conductual ha sido eficaz para disminuir la frecuencia de la ira y su expresión negativa (Beck y Fernandez, 1998), esta investigación evaluó la hipótesis de que la terapia cognitivo conductual disminuye la rumiación de la ira.

Una amenaza a cualquier estudio de intervención es que los participantes no asistan a las sesiones. Este problema es frecuente, con estudios meta-analíticos mostrando que entre el 20 y el $47 \%$ de los pacientes abandonó el tratamiento (Swift y Greenberg, 2012; Wierzbicki y Pekarik, 1993). Estos meta-análisis y diversos estudios han tratado de detectar variables que de manera frecuente caractericen a las personas que no se presentan a las sesiones (Fernandez, Salem, Swift y Ramtahal, 2015). Sin embargo, los resultados a veces han sido contradictorios y poco concluyentes: «No parece haber un único predictor que esté fuertemente asociado con discontinuar de forma temprana el tratamiento» (Salmoiraghi y Sambhi, 2010, p. 531). En cuanto al manejo de la ira, poco se ha investigado la inasistencia a las sesiones. Un estudio mostró que cuando el tratamiento fue obligatorio, debido al mandato de un tribunal, las personas asistieron a más sesiones que cuando el tratamiento fue de manera voluntaria (Dowd, Leisring y Rosenbaum, 2005). Igualmente, es necesario estudiar a las personas que concluyen los tratamientos para entender qué aspectos se asocian con la permanencia. La asistencia a un mayor número de sesiones se ha asociado con observar más características positivas en el terapeuta, el deseo del paciente de mejorar (Alcázar, 2007) y con el cumplimiento de sus expectativas (Moreno, Rozo y Cantor, 2012). Sin embargo, no encontramos estudios donde los pacientes expresen sus motivos de permanencia en tratamientos de la ira.

En síntesis, esta investigación tuvo como objetivo principal evaluar un tratamiento cognitivo conductual grupal para disminuir la rumiación, experiencia y expresión de la ira. Los objetivos secundarios son: (1) evaluar la satisfacción de los participantes con respecto a los objetivos del tratamiento, los resultados y las técnicas empleadas y, (2) identificar las razones de los participantes 
para no asistir, así como para asistir a las sesiones de tratamiento.

\section{Método}

\section{Participantes}

Se inscribieron 30 participantes (28 mujeres y 2 hombres) al tratamiento de la ira. La edad promedio fue de 38.03 ( $D T=12.66)$, en un rango de 18 a 69 años. Los participantes cumplieron estos criterios de inclusión: tener edad de 18 años o más, admitir que su ira causa daños a sí mismos o a otras personas, reconocer que tenían problemas de ira, querer disminuir dichos problemas y tener puntaje de 23 o más en la escala de Ira Rasgo (ver Instrumentos). Este puntaje indica la pertenencia al cuartil superior en la escala de Ira Rasgo, lo cual ha sido un estándar frecuente (DiGiuseppe y Tafrate, 2003) en la detección de personas con problemas de ira. Los criterios de exclusión fueron que los participantes estuvieran en alguna terapia psicológica o psiquiátrica y que experimentaran ansiedad en público (dado que el tratamiento fue grupal). En términos de ocupación, 17 personas trabajaban, ocho estudiaban, mientras que cinco estudiaban y trabajaban. Los participantes informaron que habían tenido problemas de control de la ira en un rango de 3 a 42 años $(M=18.23, D T=12.02)$.

\section{Instrumentos}

Inventario Multicultural Latinoamericano de la Expresión de la Cólera y la Hostilidad (ML-STAXI, Moscoso, 2000). Esta escala midió la experiencia, expresión y control de la ira. El ML-STAXI se creó para poblaciones latinas (Moscoso, 2000) y ha mostrado validez en población mexicana (Alcázar, Deffenbacher y Byrne, 2011). Las opciones de respuesta de escala de tipo Likert fueron de 1 (Casi nunca) a 4 (Casi siempre). La experiencia de ira se evalúa con la Escala de Ira Rasgo (10 reactivos), que se refiere a la frecuencia en que una persona siente ira en general (p.ej., «Exploto fácilmente») debido a la tendencia a sentir ira ante frustraciones específicas (p.ej., «Me enfurece que alguien cambie mis planes»). La expresión y control de la ira se evalúan con cuatro escalas. Primero, la Escala de Expresión Externa de la Ira (5 reactivos) mide conductas de ira hacia otras personas (p.ej., «Expreso mis sentimientos de furia»). Segundo, la Escala de Expresión Interna de la Ira (6 reactivos) evalúa la tendencia a suprimir la ira (p.ej., «Contengo mi enojo por muchas horas») y guardar rencores (p.ej., "Guardo rencores que no comento a nadie»). Tercero, la Escala de Control de la Ira Externa (7 reactivos) mide los intentos de controlar la propia conducta cuando la persona siente ira (p.ej., "Controlo mi forma de actuar»). Cuarto, la Escala de Control de la Ira Interna (6 reactivos) evalúa los esfuerzos por relajarse y tranquilizarse en momentos de ira (p.ej., «Trato de calmarme lo más pronto posible»). En el presente estudio, el coeficiente $\alpha$ de consistencia interna fue de .71 para la Escala de Ira Rasgo, .86 para Expresión Externa de la Ira, .77 para Expresión Interna de la Ira, .74 para Control Externo de la Ira y .89 para Control Interno de la Ira.

Escala de Rumiación de la Ira (ARS, Sukhodolsky et al., 2001). Esta escala mide la tendencia a enfocar la atención en pensamientos de experiencias presentes y pasadas en torno a la ira (Sukhodolsky et al., 2001). Las opciones de respuesta fueron de 1 (Casi nunca) a 4 (Casi siempre) y una mayor puntuación indica mayor frecuencia en la rumiación de la ira. La escala fue adaptada a población mexicana (Ortega, Alcázar-Olán, Matías, Rivera y Domínguez, 2017), y mide los mismos cuatro factores que la escala original a través de cuatro escalas: (1) La Escala de Venganza (4 reactivos) mide ideas de represalia (p.ej., «Cuando alguien me enfurece, no puedo dejar de pensar en cómo vengarme de esta persona»); (2) La Escala de Pensamientos Posteriores al Evento de Ira (6 reactivos) evalúa ideas repetitivas acerca de episodios recientes de ira (p.ej., «Después de que una discusión ha terminado, yo sigo peleando con esa persona en mi imaginación»); 3) La Escala de Entendimiento de las Causas de la Ira (4 reactivos) alude a pensar sobre las causas de eventos pasados de ira (p.ej., «Pienso en las razones por las cuales las personas me tratan mal»); y 4) La Escala de Memorias de Ira (5 reactivos) evalúa el recuerdo sobre eventos de ira (p.ej., "Permanezco pensando, por un largo tiempo, en momentos que me han causado enojo»). En el presente estudio, el coeficiente $\alpha$ de consistencia interna fue de .84 para la Escala de Venganza, .78 para Pensamientos Posteriores a la Ira, .61 para Entendimiento de las Causas de la Ira y .87 para Memorias de Ira.

Satisfacción con el tratamiento. Se crearon cinco preguntas con las cuales se indagó el grado en que los participantes se mostraron satisfechos al concluir el tratamiento. Específicamente, se les preguntó desde cero (nada satisfecho) a 10 (muy satisfecho), cómo de satisfechos estaban con: 1) los objetivos planteados en el tratamiento, 2) los resultados obtenidos con el tratamiento, 3) las técnicas de relajación, 4) las técnicas de reestructuración cognitiva y 5) los ejercicios de inoculación. En el presente estudio, el coeficiente $\alpha$ de Cronbach fue de 64 .

Preguntas sobre inasistencia y permanencia. Se indagaron los motivos de inasistencia a las sesiones, a través de dos modalidades. Primero, a quienes no asistieron 
a alguna de las sesiones, pero regresaron a la siguiente sesión, la instructora del tratamiento preguntó: ¿Por qué no asististe la sesión pasada? Segundo, a quienes no volvieron a presentarse, se les hizo la misma pregunta por llamada telefónica, correo electrónico o mensaje de texto al teléfono móvil. Los motivos de permanencia se indagaron en la sesión ocho, en la cual se pidió a los participantes responder por escrito a la pregunta: Una vez iniciado el tratamiento, ¿qué te motivó a permanecer en el mismo?

\section{Procedimiento}

El diseño de investigación fue pre-experimental con tres mediciones: pre-test, post-test y seguimiento a un mes. Este trabajo fue aprobado, en su contenido y aspectos éticos, por la Dirección de Investigación y Posgrado de la Universidad donde se llevó a cabo.

Se elaboró un cartel que difundió el tratamiento de control de la ira y se colocó en los pasillos. También se difundió por redes sociales. El cartel indicó que la inscripción debía ser directamente con la persona responsable del programa (no hubo inscripciones telefónicas o en línea). Se interesaron por la intervención 45 personas, incluyendo alumnos, exalumnos y padres de familia; varios de ellos supieron del tratamiento por medio de sus familiares que acudían a la universidad. De las 45 personas que se mostraron interesadas, 30 cumplieron los criterios de inclusión y firmaron el consentimiento informado. La carta de consentimiento incluyó los siguientes puntos: 1) la duración del tratamiento, el cual fue de nueve sesiones y una reunión de seguimiento, un mes posterior a la novena sesión, 2) en qué consistió el tratamiento, 3) el compromiso de asistir a todas las sesiones, 4) la solicitud del permiso para contactarles por teléfono o correo electrónico, para preguntarles los motivos de inasistencia, en caso de haberlas y 5) la aclaración de que el tratamiento era voluntario y podían desertar, sin ninguna penalización.

A las 30 personas inscritas se les dividió en dos grupos para impartirles el tratamiento. Uno tuvo 14 personas y el otro 13 (tres personas no asistieron a ninguna sesión). El criterio para el tamaño de los grupos fue en cumplimiento del manual del terapeuta (Deffenbacher y McKay, 2000a) para impartir un tratamiento grupal de la ira: «Establezca grupos de aproximadamente seis a doce personas. Esto permite un grupo suficientemente grande para la interacción, pero suficientemente pequeño para atender preocupaciones individuales específicas e individualizar la intervención» (Deffenbacher y McKay, 2000a, p. 180).

\section{Intervención}

El manual de tratamiento cognitivo conductual de la ira (Deffenbacher y McKay, 2000a) indicó el contenido y actividades de cada sesión. La primera sesión fue introductoria al tratamiento. En la segunda y tercera sesión se enseñaron las siguientes técnicas de relajación: con una imagen específica, respiración profunda, muscular progresiva, con una palabra tranquilizante y sin tensar los músculos. Estas técnicas se encuentran en Deffenbacher y McKay (2000a, pp. 75-80 y 88-89). La sesión cuatro fue sobre reestructuración cognitiva. Las sesiones cinco a nueve fueron sobre inoculación de la ira, en la cual las personas imaginaron la situación de ira, sintieron dicha emoción y luego usaron habilidades de relajación y reestructuración cognitiva para disminuir la ira. Las inoculaciones se hicieron al menos tres veces por cada situación de ira. Conforme avanzaron las sesiones, se emplearon situaciones de ira cada vez más intensa.

A cada persona se le entregó en la primera sesión un «Manual del Participante», el cual se basó en el Manual del Cliente para el Tratamiento de la Ira (Deffenbacher y McKay, 2000b). Este manual incluyó las técnicas y las tareas de cada sesión.

Las instructoras del tratamiento fueron dos alumnas del último semestre de la licenciatura en psicología, una para cada grupo. Dichas alumnas leyeron el Manual del Terapeuta (Deffenbacher y McKay, 2000a) y, a modo de ensayo, impartieron las sesiones al responsable del programa, quien también supervisó las sesiones con los participantes.

\section{Análisis estadístico}

Se usaron tres criterios para evaluar el tratamiento. Primero, se buscaron diferencias estadísticamente significativas con la prueba de Wilcoxon. Segundo, se evaluó el tamaño del efecto para conocer la magnitud de los cambios. Este efecto se calculó con la fórmula $r=\mathrm{Z} / \sqrt{ } \mathrm{N}$ (Rosenthal, 1991), donde valores entre .00 y .30 son bajos, entre .30 y .50 moderados, y arriba de .50 fuertes. Tercero, se evaluó el tratamiento de forma individual, en función del cambio clínico que logró cada participante, uno por uno (Jacobson y Truax, 1991).

El cambio clínico se midió a partir de los cambios en el post-test y seguimiento a un mes de cada participante. Este procedimiento se siguió con cada una de las variables medidas con el ML-STAXI y la Escala de Rumiación de la Ira. Con base en Jacobson y Truax (1991), se asignó a cada participante, en cada variable, uno de los siguientesestatus: recuperado (R), mejorado pero no recuperado (M) y no mejorado (NM). El término recupera- 
do se refirió a cumplir dos aspectos: uno es que el participante alcanzó el punto de corte, lo cual indicó que la persona ya no pertenece a la población disfuncional sino a la población normativa, y el otro fue que el participante alcanzó el índice de cambio confiable, lo cual indicó que el cambio no se debió a imprecisiones en el instrumento de medición (Jacobson y Truax, 1991, p. 14). El término mejorado pero no recuperado, indicó que el participante no alcanzó el punto de corte, pero sí el índice de cambio confiable. Finalmente, el término no mejorado se refirió a que el participante no alcanzó el punto de corte, ni el índice de cambio confiable.

Los puntos de corte, esto es, los puntajes que dividieron a la población disfuncional de la normativa, se obtuvieron con el apoyo del programa MacRCI: Reliable Change Index (Watkins, 2006), el cual se basó en Jacobson y Truax (1991). De dicho programa se obtuvo también el índice de cambio confiable. Para el uso del programa se usaron datos de muestras clínicas y normativas. Los datos de la muestra clínica para el ML-STAXI se tomaron de 142 pacientes (33 hombres y 109 mujeres, $M e$ dia de edad $=38.49, D T=13.54)$, pertenecientes a otra investigación, que buscaron tratamiento de control de la ira y que tenían como característica esencial alta ira rasgo, criterio estándar en la detección de personas con problemas de ira (DiGiuseppe y Tafrate, 2003). Los datos normativos del ML-STAXI se tomaron de otro estudio (Alcázar-Olán, Deffenbacher, Hernández-Guzmán, y de la Chaussée, 2014). Para la escala de rumiación, los datos se tomaron de Ortega et al., (2017). Los datos fueron normativos en ambas escalas en el sentido de que no incluían personas disfuncionales, lo cual obedeció a la recomendación de Jacobson, Roberts, Berns, y McGlinchey (1999). En particular, el percentil 75 en el puntaje total de rumiación y en la escala de ira rasgo fue el criterio que separó a la población funcional de la disfuncional.

\section{Resultados}

\section{Análisis preliminares}

Ambos grupos fueron equivalentes en todos sus puntajes antes del tratamiento, lo cual se reflejó en ausencia de diferencias significativas en las nueve variables medidas, $U$ de Mann Whitney $=70.0-87.5, p \mathrm{~s}>.05$.

\section{Asistencia a las sesiones}

Unas personas asistieron a más sesiones que otras. Específicamente, tres personas no asistieron a ninguna sesión, una persona fue a una sesión, tres personas a tres sesiones, dos a cuatro sesiones, una a cinco sesiones, tres a siete sesiones, nueve a ocho sesiones y ocho a las nueve sesiones. A quienes asistieron siete o más sesiones $(n=20)$, se les consideró como personas de alta asistencia; de todas ellas se obtuvo información del post-test o seguimiento. En cambio, quienes asistieron menos de siete sesiones $(n=10)$, denominados a partir de aquí personas de baja asistencia, obtuvimos información de post-test o seguimiento de sólo cinco de ellos. De cinco personas de baja asistencia no se consiguió ningún posttest, ni seguimiento. Dado que el objetivo fue evaluar los efectos del tratamiento, se analizó la información en dos grupos: a quienes tuvieron alta versus los que tuvieron baja asistencia.

\section{Ira rasgo, control y expresión de la ira}

Como resultado de la intervención, se observaron mejoras estadísticamente significativas únicamente en el grupo de alta asistencia, tanto en el post-test como en el seguimiento (Tabla 1). El grupo de baja asistencia no mejoró de forma estadísticamente significativa en ninguna variable. Sin embargo, estos resultados podrían estar sesgados debido a que el grupo de alta asistencia tuvo más participantes que el de baja asistencia, lo cual aumentó el poder estadístico para encontrar diferencias significativas. Para evitar este sesgo y lograr otra perspectiva, se calculó también el tamaño del efecto. Como resultado, ambos grupos, tanto el de alta como el de baja asistencia, mejoraron en la misma proporción, lo cual se reflejó en tamaño del efecto fuerte, tanto en el post-test como en el seguimiento (Tabla 1).

Después de los análisis grupales, se analizaron los cambios individuales. En todas las variables, se evaluó el cambio clínico (Jacobson y Truax, 1991) con los resultados del seguimiento a un mes. Sin embargo, cuando esa información no estuvo disponible (debido a que no se pudo contactar al participante), se consideró únicamente el resultado del post-test. Los resultados de los participantes de alta y baja asistencia se informan por separado. Con respecto a los de alta asistencia, se observó que en la variable de ira rasgo (Tabla 2), de los 20 participantes, 12 alcanzaron el estatus de recuperado, uno el estatus de mejorado pero no recuperado, y siete el estatus de no mejorado.

En las demás variables se encontró que los puntajes de algunas personas, en el pre-test, estaban dentro del punto de corte considerado como normal. Observamos que estas personas, que al inicio estaban «normales», arrojarían información errónea en los análisis. Prueba de ello fue el participante cinco, quien obtuvo 20 en el puntaje pre-test en la variable de control externo de la ira, y dicho puntaje estuvo arriba del punto de corte, lo cual indicó normalidad. 
Tabla 1. Grupos de alta $(n=20)$ y baja asistencia $(n=5)$, cambio en variables de ira, rumiación y tamaño del efecto

\begin{tabular}{|c|c|c|c|c|c|c|c|c|c|c|c|}
\hline \multirow{2}{*}{ Variables } & \multirow{2}{*}{$\begin{array}{c}\text { Tipo de } \\
\text { asistencia }\end{array}$} & \multicolumn{2}{|c|}{ Pre-test } & \multicolumn{2}{|c|}{ Post-test } & \multicolumn{2}{|c|}{ Seguimiento } & \multirow{2}{*}{$\begin{array}{c}\text { Prueba } \\
\text { Wilcoxon }\end{array}$} & \multirow{2}{*}{$\begin{array}{c}\begin{array}{c}\text { Prueba } \\
\text { Wilcoxon }\end{array} \\
\text { Seg. }\end{array}$} & \multirow{2}{*}{$\begin{array}{c}T E \\
\text { Post-test }\end{array}$} & \multirow{2}{*}{$\begin{array}{l}\text { TE } \\
\text { Seg. }\end{array}$} \\
\hline & & $M$ & $D T$ & $M$ & $D T$ & $M$ & $D T$ & & & & \\
\hline \multirow[t]{2}{*}{ Ira rasgo } & Alta & 29.6 & 4.3 & 20.8 & 5.0 & 17.7 & 3.1 & $-3.9 * * *$ & $-3.1 * *$ & .87 & .88 \\
\hline & Baja & 30.0 & 3.9 & 22.0 & 2.9 & 17.6 & 5.0 & -1.8 & -1.6 & .91 & .92 \\
\hline \multirow[t]{2}{*}{ Control externo de la ira } & Alta & 14.2 & 3.5 & 19.1 & 2.9 & 22.9 & 3.9 & $-3.6 * * *$ & $-2.9 * *$ & .80 & .83 \\
\hline & Baja & 14.1 & 1.7 & 16.7 & 3.3 & 18.0 & 2.6 & -1.8 & -1.6 & .91 & .92 \\
\hline \multirow[t]{2}{*}{ Ira externa } & Alta & 14.3 & 3.9 & 11.5 & 3.2 & 10.1 & 2.0 & $-2.7 * *$ & $-2.8 * *$ & .59 & .82 \\
\hline & Baja & 13.5 & 4.5 & 12.7 & 2.9 & 11.0 & 1.7 & -0.2 & -1.6 & .09 & .92 \\
\hline \multirow[t]{2}{*}{ Ira interna } & Alta & 13.7 & 5.3 & 10.0 & 3.1 & 9.5 & 3.5 & $-3.3 * *$ & $-2.8 * *$ & .73 & .82 \\
\hline & Baja & 14.8 & 3.3 & 13.2 & 4.7 & 12.6 & 1.5 & 0.0 & -1.1 & .00 & .62 \\
\hline \multirow[t]{2}{*}{ Control interno de la ira } & Alta & 14.1 & 4.7 & 19.6 & 3.6 & 21.2 & 3.2 & $-3.1 * *$ & $-2.8 * *$ & .70 & .80 \\
\hline & Baja & 12.4 & 3.6 & 17.7 & 1.5 & 17.3 & 5.0 & -1.8 & -1.6 & .92 & .92 \\
\hline \multirow[t]{2}{*}{ Venganza } & Alta & 7.7 & 3.4 & 5.9 & 1.3 & 5.1 & 1.1 & $-2.1 *$ & $-2.1 *$ & .46 & .61 \\
\hline & Baja & 9.2 & 1.8 & 6.5 & 1.9 & 5.3 & 1.5 & -1.5 & -1.6 & .73 & .94 \\
\hline \multirow{2}{*}{$\begin{array}{l}\text { Pensamientos posteriores } \\
\text { de ira }\end{array}$} & Alta & 15.4 & 4.7 & 10.3 & 2.5 & 9.1 & 1.8 & $-3.2 * *$ & $-2.9 * *$ & .72 & .84 \\
\hline & Baja & 14.0 & 3.8 & 12.0 & 3.3 & 6.3 & 0.5 & -1.5 & -1.6 & .73 & .92 \\
\hline \multirow[t]{2}{*}{ Causas de la ira } & Alta & 10.2 & 2.6 & 9.1 & 2.9 & 7.2 & 2.0 & -1.3 & $-2.7 * *$ & .29 & .77 \\
\hline & Baja & 10.7 & 2.7 & 8.7 & 2.5 & 5.6 & 2.8 & -1.6 & -1.6 & .80 & .92 \\
\hline \multirow[t]{2}{*}{ Memorias de ira } & Alta & 13.7 & 3.6 & 10.0 & 3.1 & 8.4 & 1.7 & $-3.2 * *$ & $-3.1 * *$ & .71 & .88 \\
\hline & Baja & 12.3 & 3.2 & 10.0 & 4.3 & 6.0 & 2.6 & -0.7 & -1.6 & .36 & .92 \\
\hline
\end{tabular}

Nota. Seg = Seguimiento; TE: Tamaño del efecto. ${ }^{*} p<.05, * * p<.01, * * * p<.001$.

Tabla 2. Número de participantes de alta $(n=20)$ y baja asistencia $(n=5)$ y su estatus

\begin{tabular}{|c|c|c|c|c|c|}
\hline \multirow{2}{*}{ Variables } & \multirow{2}{*}{$\begin{array}{c}\text { Tipo de } \\
\text { asistencia }\end{array}$} & \multicolumn{4}{|c|}{ Estatus después del tratamiento } \\
\hline & & Recuperados & Mejorados & No mejorados & Normales* \\
\hline \multirow[t]{2}{*}{ Ira rasgo } & Alta & 12 & 1 & 7 & 0 \\
\hline & Baja & 3 & 0 & 2 & 0 \\
\hline \multirow[t]{2}{*}{ Control externo } & Alta & 6 & 0 & 8 & 6 \\
\hline & Baja & 1 & 0 & 4 & 0 \\
\hline \multirow[t]{2}{*}{ Expresión externa } & Alta & 4 & 0 & 12 & 4 \\
\hline & Baja & 2 & 0 & 1 & 2 \\
\hline \multirow[t]{2}{*}{ Expresión interna } & Alta & 4 & 0 & 7 & 9 \\
\hline & Baja & 0 & 0 & 3 & 2 \\
\hline \multirow[t]{2}{*}{ Control interno } & Alta & 6 & 0 & 6 & 8 \\
\hline & Baja & 3 & 0 & 1 & 1 \\
\hline \multirow[t]{2}{*}{ Venganza } & Alta & 7 & 0 & 0 & 13 \\
\hline & Baja & 2 & 0 & 0 & 3 \\
\hline \multirow[t]{2}{*}{ Pensamientos posteriores a la ira } & Alta & 11 & 0 & 1 & 8 \\
\hline & Baja & 4 & 0 & 0 & 1 \\
\hline \multirow[t]{2}{*}{ Causas de la ira } & Alta & 6 & 1 & 3 & 10 \\
\hline & Baja & 4 & 0 & 1 & 0 \\
\hline \multirow[t]{2}{*}{ Memorias de la ira } & Alta & 9 & 0 & 2 & 9 \\
\hline & Baja & 2 & 0 & 0 & 3 \\
\hline
\end{tabular}

*Normal se refiere a que los participantes, antes del tratamiento, ya pertenecían a un rango no disfuncional.

El resultado del análisis del cambio clínico mostró que este participante quedaría en el estatus de «no mejorado». No tiene sentido considerar «no mejorado» a alguien que ya era normal. Para evitar estas interpretaciones erróneas, se incluyó otra categoría en los resultados: la de personas que en el pre-test tenían puntajes normales (Tabla 2). 
En la variable de control externo de la ira (Tabla 2), seis personas se recuperaron, ocho no mejoraron y seis tenían puntaje normal en el pre-test. En expresión externa de la ira, cuatro personas se recuperaron, 12 no mejoraron y cuatro fueron normales en el pre-test. En expresión interna de la ira, cuatro personas se recuperaron, siete no mejoraron y nueve eran normales en el pre-test. En el control interno de la ira, seis participantes se recuperaron, seis no mejoraron y ocho eran normales en el pre-test. En síntesis, de los 20 participantes que asistieron a siete o más sesiones, 17 se beneficiaron en una o más variables del ML-STAXI (lograron el estatus de recuperado o mejorado). Tres de ellos no se beneficiaron o tenían puntajes normales en el pre-test. Las variables donde hubo mayor cambio fueron ira rasgo y control de la ira interna y externa. Con respecto a las cinco personas que asistieron menos de siete sesiones, y de las cuales se tiene el post-test o seguimiento, se encontró que todas mejoraron en al menos una y como máximo en dos variables del ML-STAXI.

\section{Rumiación}

La mejora en rumiación se analizó desde dos perspectivas: por cada variable de rumiación y por el número de variables en que las personas se recuperaron. Con respecto a las variables de rumiación, se encontró que, de las 20 personas de alta asistencia, en ideas de venganza, siete participantes se recuperaron y 13 eran normales en el pretest. En pensamientos posteriores a la ira, 11 personas se recuperaron, una no mejoró y ocho eran normales en el pre-test. En entendimiento de causas de la ira, seis se recuperaron, una mejoró, tres no mejoraron y 10 eran normales en el pre-test. En memorias de ira, nueve se recuperaron, dos no mejoraron y nueve eran normales en el pre-test.

Con respecto al número de variables donde hubo recuperación, de las 20 personas de alta asistencia, cuatro alcanzaron el estatus de recuperado en una variable de rumiación, dos personas en dos variables, tres personas en tres variables y cuatro personas en las cuatro variables. Esto es, 13 personas se recuperaron en una o más variables. Cinco personas tuvieron puntajes normales en el pre-test y dos no se beneficiaron. Las variables con mayores cambios fueron pensamientos posteriores al evento de ira y memorias de ira. Las personas que asistieron menos de siete sesiones mejoraron en al menos una de las cuatro variables de rumiación.

\section{Satisfacción}

En la escala de 0 a 10, los grupos de alta y baja asistencia se mostraron satisfechos con los objetivos plan- teados en el tratamiento $(M=9.60$ y $9.66, D T=0.59$ y 0.57 , respectivamente), los resultados alcanzados $(M=$ 9.45 y $8.66, D T=0.68$ y 1.15 , respectivamente) y las técnicas de relajación $(M=9.50$ y $9.00, D T=0.60$ y 1.00 , respectivamente). Sin embargo, el grupo de alta asistencia estuvo más satisfecho que el de baja en las técnicas de reestructuración cognitiva $(M=9.65$ y 6.33 , $D T=0.58$ y 5.50 , respectivamente) y los ejercicios de inoculación $(M=8.95$ y $4.50, D T=1.09$ y 6.36 , respectivamente). Ninguna de las diferencias entre los grupos fue estadísticamente significativa, $U$ de Mann Whitney = $9-29, p \mathrm{~s}>.05$. La mayoría de los participantes, especialmente los de alta asistencia, expresaron espontáneamente que los ejercicios de inoculación, aunque fueron útiles, también fueron muy cansados, repetitivos y largos.

\section{Motivos de inasistencia}

Las personas de baja y alta asistencia mostraron distintos motivos para no asistir a las sesiones. Las personas de baja asistencia no se presentaron, en primer lugar, por motivos escolares ( 5 personas), como realizar tareas escolares y, en segundo lugar, por motivos laborales (2 personas), como el exceso de trabajo. Los demás no se presentaron por problemas de salud (1 persona), motivos personales (1 persona) y de un participante no se consiguió información.

En cuanto a las personas que asistieron siete y ocho sesiones, sus motivos de inasistencia fueron, en primer lugar, problemas de salud ( 5 personas), en segundo lugar, por asuntos laborales ( 3 personas), en tercer lugar, por motivos personales ( 3 personas) y en cuarto lugar por motivos escolares ( 1 persona).

\section{Motivos de asistencia}

A la pregunta de qué les motivó a permanecer en el tratamiento, algunos participantes expresaron más de una idea en una sola respuesta, p.ej., «me motivó el deseo de cambiar y me di cuenta de que el tratamiento me servía». Para el análisis de resultados, ejemplos como este se dividieron en dos unidades de respuesta: «Me motivó el deseo de cambiar» y «Me di cuenta de que el tratamiento me servía». Los participantes respondieron con al menos una unidad de respuesta y como máximo cuatro. Específicamente, 10 personas dieron una sola respuesta, seis personas dos, dos personas tres y dos personas cuatro (Tabla 3 ).

Con base en el contenido de cada respuesta, los dos primeros autores crearon categorías para agruparlas. Las categorías fueron: 1) Resultados alcanzados con el tratamiento, es decir, respuestas que mostraron logros o ga- 
nancias en la vida personal y en el manejo de la ira (p.ej., «Las técnicas me funcionaron para controlar mi enojo»); 2) Logro de metas internas, es decir, alcanzar objetivos intrínsecos asociados a las expectativas del tratamiento, (p.ej., «Tenía ganas de ser mejor persona» y 3) Aspectos externos, es decir, lo agradable del tratamiento pero que no refleja directamente resultados positivos (p.ej., «El atinado comentario de los demás participantes») y 4) Otros, donde se incluyeron las respuestas que no entraban en las tres categorías anteriores (p.ej., «Tenía curiosidad»).

Como resultado (Tabla 3), las categorías que predominaron para asistir a varias sesiones fueron los resultados alcanzados con el taller (17 respuestas) y el logro de metas internas (14 respuestas). Otras categorías fueron menos frecuentes, como los aspectos externos del taller (4 respuestas) y otras razones (1 respuesta).

\section{Discusión}

Los resultados de este trabajo apoyan parcialmente la hipótesis de que la terapia cognitivo conductual disminuye la rumiación de la ira. De 20 personas con alta asistencia, 13 lograron cambio clínico (Jacobson y Truax, 1991), es decir, el estatus de recuperado, principalmente en pensamientos posteriores a la ira y en memorias de ira. Cinco tuvieron puntajes normales en el pre-test y dos no se beneficiaron. Los resultados apoyan que tomar el tratamiento completo benefició a la mayoría de los participantes, lo cual sugiere cierta eficacia de la intervención cognitivo conductual (Deffenbacher y McKay, 2000a) para problemas de rumiación. Desde una perspectiva global, incluyendo a las variables medidas por el ML-STAXI y la escala de rumiación, de las personas de

Tabla 3. Motivos de los participantes de alta asistencia para permanecer en el tratamiento $(n=20)$

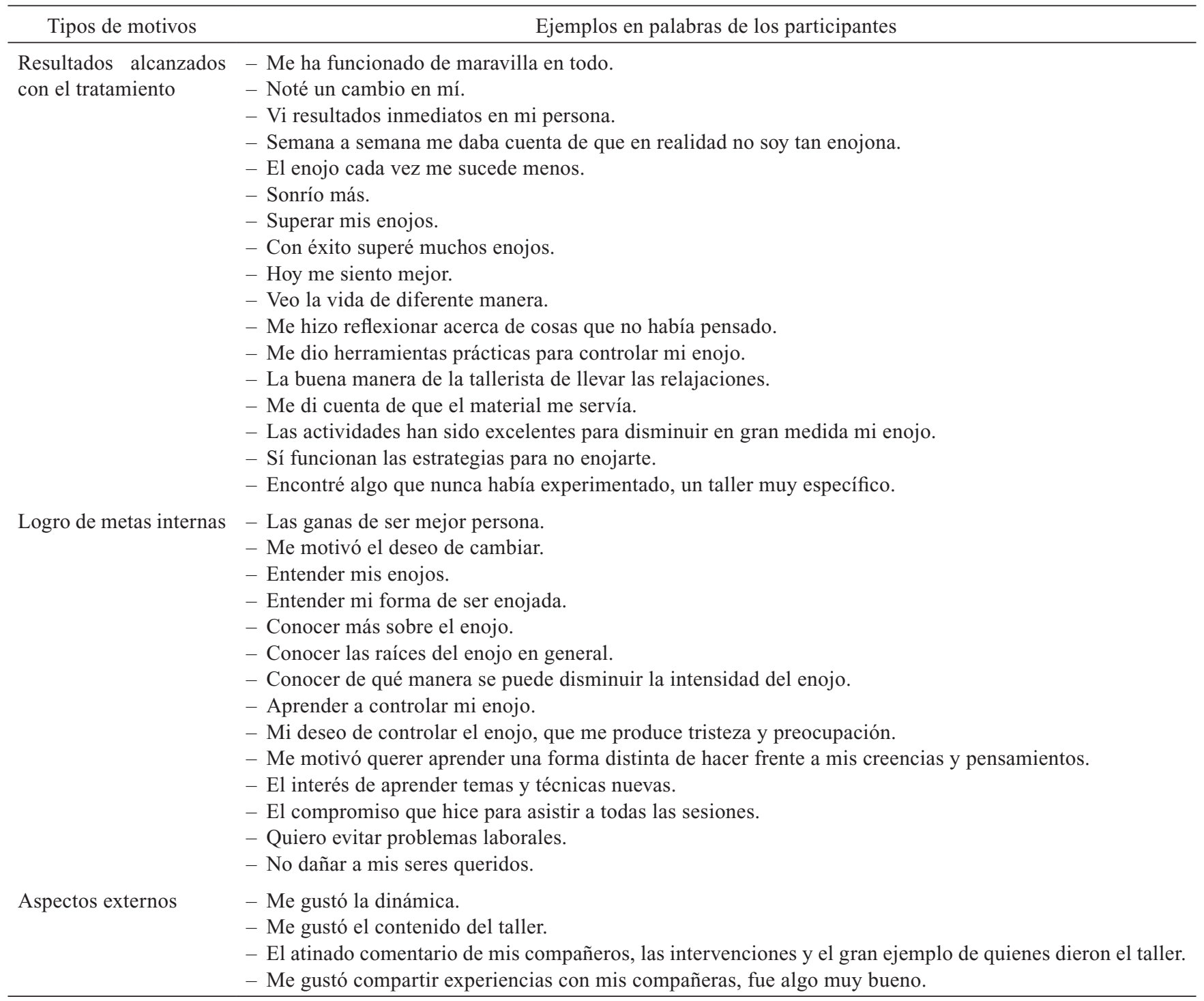


alta asistencia, tres de ellas no mejoraron en ninguna de las nueve variables, mientras que 17 personas mejoraron en al menos una variable y como máximo en nueve. En el ML-STAXI, la mejora se reflejó principalmente en ira rasgo, control externo e interno de la ira.

Las personas que asistieron menos de siete sesiones cambiaron principalmente en pensamientos posteriores a la ira, causas de la ira y memorias de ira. Sin embargo, estos resultados reflejan el estatus de sólo cinco de 10 personas. No se pudo conseguir el post-test o seguimiento de los otros cinco participantes, por lo cual se desconoce su estatus. En consecuencia, tener sólo a la mitad de los participantes de baja asistencia impide conclusiones firmes con respecto a sus cambios. Los participantes de alta asistencia se mostraron satisfechos con los objetivos del tratamiento, los resultados alcanzados, las técnicas de relajación y la reestructuración cognitiva. Sin embargo, expresaron que los ejercicios de inoculación eran repetitivos, tediosos, largos y cansados. Este cansancio probablemente aumentó debido a que varias veces, de la sesión cinco a nueve, se usó la inoculación. Aunque no se investigó directamente, es posible que el tedio de las inoculaciones pudo influir en las inasistencias de algunos participantes.

El grupo de baja asistencia se ausentó principalmente por motivos escolares, seguido por motivos laborales y problemas de salud. En cambio, el grupo de alta asistencia, cuando no asistió a las sesiones, fue en primer lugar por problemas de salud, seguido por asuntos laborales y finalmente por motivos escolares. Esto se asocia con la edad y la ocupación de los participantes. Los jóvenes universitarios (seis de 10 personas del grupo de baja asistencia) son los que tuvieron más inasistencias. En cambio, las personas cuya ocupación es sólo trabajar son las que asisten a un mayor número de sesiones. Se observó que 14 de 20 personas sólo trabajan y asistieron a siete o más sesiones. Estos resultados concuerdan con un estudio donde una gran proporción de las personas que deja el tratamiento son jóvenes (Swift y Greenberg, 2012). La inasistencia de los jóvenes universitarios quizás se deba a que ellos tienen muchas tareas escolares y además se les indicó en la carta de consentimiento que podían desertar sin ninguna penalización.

En cuanto a los motivos para permanecer en el tratamiento, los resultados muestran que los principales motivos para completar siete o más sesiones son los resultados alcanzados, es decir, las personas vieron resultados inmediatos y disminuyeron su ira. Esto concuerda con otro estudio donde el cumplimiento de las expectativas se asoció con mayor asistencia a las sesiones (Moreno et al., 2012). Otro aspecto igualmente relevante para completar las sesiones fue el logro de metas internas. En par- ticular, el compromiso de asistir a todas las sesiones y el interés de aprender sobre la ira. Esto sugiere que notar logros palpables y tener motivación intrínseca contribuyen a seguir en el tratamiento. Investigaciones futuras podrían evaluar el grado en que existe motivación intrínseca antes de iniciar el tratamiento de control de la ira para detectar participantes que podrían abandonar el tratamiento.

Este estudio presenta limitaciones. En primer lugar, la mayoría de los participantes fueron mujeres. Esto probablemente se explica porque es más fácil que las mujeres, a diferencia de los hombres, acepten y busquen ayuda psicológica (Wend y Shafer, 2015). En consecuencia, los resultados encontrados se limitan, predominantemente, al sexo femenino. En segundo lugar, la inexistencia de grupo control impide asegurar que los resultados positivos se deben al tratamiento. En tercer lugar, varios participantes no completaron el seguimiento a un mes (en concreto, sólo se consiguieron 12 mediciones de 20 posibles en el grupo de alta asistencia, y tres de 10 en el grupo de baja asistencia). En consecuencia, el panorama de los resultados es sólo parcial: no incluye a todos los participantes y el seguimiento es muy corto (un mes), lo cual impide conclusiones sólidas. Sin embargo, consideramos que el tamaño de la muestra (20 personas de alta asistencia y 10 de baja asistencia) permite observar una tendencia en los resultados, especialmente en el grupo de alta asistencia. Futuras investigaciones deberían incluir estrategias que hayan mostrado ser útiles para lograr que las personas asistan a las sesiones y se logre el seguimiento de todos o de la mayoría, aún si no completan las sesiones.

En conclusión, el tratamiento cognitivo conductual fue eficaz en la mayoría de los participantes. Mejoraron en la expresión, control y rumiación de la ira. Quienes tuvieron más inasistencias fue por motivos escolares. Las personas que completaron el tratamiento informaron resultados positivos y alta motivación interna.

\section{Conflicto de intereses}

Los autores manifiestan que no existe conflicto de intereses.

\section{Referencias}

Alcázar, R. J. (2007). Expectativas, percepción del paciente hacia su terapeuta y razones para asistir a dos o más sesiones. Salud Mental, 30(5), 55-62.

Alcázar, R. J., Deffenbacher, J. L., \& Byrne, Z. S. (2011). Assessing the factor structure of the anger expression inventory (ML-STAXI) in a Mexican sample. International Journal of Psychology and Psychological Therapy, 11(2), 307-318. 
Alcázar-Olán, R. J., Deffenbacher, J. L., Hernández-Guzmán, L. \& de la Chaussée, M. E. (2014). The angry thoughts scale: Initial development in a Mexican sample. International Journal of Cognitive Therapy, 7, 373-388.

Beck, R. \& Fernandez, E. (1998). Cognitive-behavioral therapy in the treatment of anger: A meta-analysis. Cognitive Therapy and Research, 22, 63-74.

Catilho, P., Xavier, A., Pinto-Gouveia, J., Costa, T. (2015). When the «threat system» is switched on: The impact of anger and shame on paranoia. Revista de Psicopatología y Psicología Clínica, 20 (3), 199-208.

Cárdenas, M. y Arancibia, H. (2014). Potencia estadística y cálculo del tamaño del efecto en $\mathrm{G}^{*}$ power: Complementos a las pruebas de significación estadística y su aplicación en psicología. Salud y Sociedad, 5(2), 210-224.

Carter, S. L. (2010). The social validity manual: A guide to subjective evaluation of behavior interventions in applied behavior analysis. London: Academic Press.

Deffenbacher, J. L. (1992). Trait anger: Theory, findings, and implications. En C.D. Spielberger \& J.N. Butcher (Eds.), Advances in personality assessment (Vol. 9, pp. 177-201). Hillsdale, NJ: Lawrence Erlbaum Associates.

Deffenbacher, J. L. \&McKay, M. (2000a). Overcoming situational and general anger: A protocol for the treatment of anger based on relaxation, cognitive restructuring, and coping skills training (therapist protocol). Oakland, CA: New Harbinger.

Deffenbacher, J. L. \& McKay, M. (2000b). Overcoming situational and general anger: A protocol for the treatment of anger based on relaxation, cognitive restructuring, and coping skills training (client manual). Oakland, CA: New Harbinger.

Deffenbacher, J. L. (2006). Evidence for effective treatment of anger-related disorders. En E. L. Feindler (Ed.), Anger-related disorders: A practitioner's guide to comparative treatments (pp. 43-69). New York, NY: Springer Publishing Company.

Denson, T. F., Fabiansson, E. C., Creswell, J. D., \& Pedersen, W. C. (2009). Experimental effects of rumination styles on salivary cortisol responses. Motivation and Emotion, 33, 42-48.

DiGiuseppe, R. \& Tafrate, R. C. (2003). Anger treatment for adults: A meta-analytic review. Clinical Psychology: Science and Practice, 10, 70-84.

Dowd, L. S., Leisring, P. A., \& Rosenbaum, A. (2005). Partner aggressive women: Characteristics and treatment attrition. Violence and Victims, 20, 219-233.

Edmonson, C. B. \& Conger, J. C. (1996). A review of treatment efficacy for individuals with anger problems: Conceptual, assessment, and methodological issues. Clinical Psychology Review, 16, 251-275.

Fernandez, E., Salem, D., Swift, J. K., \& Ramtahal, N. (2015). Meta-analysis of dropout from cognitive behavioral therapy: Magnitude, timing, and moderators. Journal of Consulting and Clinical Psychology, 83, 1108-1122.

Gorenstein, E. E., Tager, F. A., Shapiro, P. A., Monk, C., \& Sloan, R. P. (2007). Cognitive-behavior therapy for reduction of persistent anger. Cognitive and Behavioral Practice, 41, 168-184.

Izadpanah, S., Schumacher, M., \& Barnow, S. (2017). Anger rumination mediates the relationship between reinforcement sensitivity and psychopathology: Results of a 5-year longitudinal study. Personality and Individual Differences, 110, 4954.
Jacobson, N. S., Roberts, L. J., Berns, S. B., \& McGlinchey, J. B. (1999). Methods for defining and determining the clinical significance of treatment effects: Description, application, and alternatives. Journal of Consulting and Clinical Psychology, 67, 300-307.

Jacobson, N. S. \& Truax, P. (1991). Clinical significance: A statistical approach to defining meaningful change in psychotherapy research. Journal of Consulting and Clinical Psychology, 59, $12-19$.

Kassinove, H. \& Tafrate, R. C. (2006). Anger-related disorders: Basic issues, models, and diagnostic considerations. En E. L. Feindler (Ed.), Anger-related disorders: A practitioner's guide to comparative treatments (pp. 1-27). New York: Springer publishing company.

Moreno, J. H, Rozo, M., \& Cantor, I. (2012). Permanencia y abandono terapéutico en un centro de servicios psicológicos. Psychologia, Avances de la Disciplina, 6, 24-34.

Moscoso, M. (2000). Estructura factorial del inventario multicultural latinoamericano de la expresión de la cólera y la hostilidad. Revista Latinoamericana de Psicología, 32(2), 321-343.

Novaco, R. W. (1976). The treatment of chronic anger through cognitive and relaxation controls. Journal of Consulting and Clinical Psychology, 44, 681.

Novaco, R. W. (1978). Anger and coping with stress: Cognitive-behavioral interventions. En J. P. Foreyt y D. Rathjen (Eds.), Cognitive behavior therapy: Research application (pp. 135-173). New York, NY: Plenum Press.

Ortega, N., Alcázar-Olan, R., Matías, O. M., Rivera, A., \& Domínguez, A. (2017). Anger rumination scale: Validation in Mexico. The Spanish Journal of Pscychology, 20, 1-9.

Rosenthal, R. (1991). Meta-analytic procedures for social research (2nd ed.). Newbury Park, CA: Sage.

Salmoiraghi, A. \& Sambhi, R. (2010). Early termination of cognitive-behavioural interventions: Literature review. The Psychiatrist, 34, 529-532.

Schum, J. L., Jorgensen, R. S., Verhaeghen, P., Sauro, M., \& Thibodeau, R. (2003). Trait anger, anger expression, and ambulatory blood pressure: A meta-analytic review. Journal of Behavioral Medicine, 26, 395-415.

Spielberger, C., D. \& Reheiser, E. C. (2009). Assessment of emotions: Anxiety, anger, depression, and curiosity. Applied Psychology: Health and Well-being, 1, 271-302.

Stimmel, D. T., Rayburg, J., Waring, W., \& Raffeld, P. M. (2005). The relation of internalized and trait anger to psychopathology. Counseling and Clinical Psychology Journal, 2(3), 112123.

Sukhodolsky, D. G., Golub, A., \& Cromwell, E. N. (2001). Development and validation of the anger rumination scale. Personality and Individual Differences, 31, 689-700.

Swift, J. K. \& Greenberg, R. P. (2012). Premature discontinuation in adult psychotherapy: A meta-analysis. Journal of Consulting and Clinical Psychology, 80, 547-559.

Takebe, M., Takahashi, F., \& Sato, H. (2016). Anger rumination as a risk factor for trait anger and anger in: A longitudinal study. Journal of Personality and Individual Differences, 101, 451455.

Wang, X., Yang, L., Yang, J., Wang, P., \& Lei, L. (2017). Trait anger and cyberbullhying among young adults: A moderated 
mediation model of moral disengagement and moral identity. Computers in Human Behavior, 73, 519-426.

Watkins, M. W. (2006). Mac RCI: Reliable Change Index. Disponible en http://edpsychassociates.com/Watkins3.html

Wend, D. \& Shafer, K. (2015). Gender and attitudes about mental health help seeking: Results from national data. Health \& Social Work, 41, 20-28.
Wierzbicki, M. \& Pekarik, G. (1993). A meta-analysis of psychotherapy dropout. Professional Psychology: Research and Practice, 24, 190-195.

Wilkowski, B. M. \& Robinson, M. D. (2007). Keeping one's cool: Trait anger, hostile thoughts, and the recruitment of limited capacity control. Personality and Social Psychology Bulletin, 33, 1201-1213. 
